\title{
Cardiac Myeloperoxidase Activity Is Elevated in Hypertensive Pregnant Rats
}

\author{
Ming-lin ZHU (朱明林) ${ }^{1}$, Jin-ping ZHAO (赵金平) ${ }^{1 \#}$, Ning CUI (崔 凝 $)^{2}$, Victor H. Gonçalves-Rizzi ${ }^{3}$, Jose S. Possomato-Vieira ${ }^{3}$, \\ Regina A. Nascimento ${ }^{3}$, Carlos A. Dias-Junior ${ }^{3 \#}$ \\ ${ }^{1}$ Department of Thoracic and Cardiovascular Surgery, Zhongnan Hospital, Wuhan University, Wuhan 430071, China \\ ${ }^{2}$ Department of Gastroenterology, Renmin Hospital of Wuhan University, Wuhan 430060, China \\ ${ }^{3}$ Department of Pharmacology, Biosciences Institute of Botucatu, Sao Paulo State University (UNESP), Botucatu 18618681, Brazil
}

(C) Huazhong University of Science and Technology and Springer-Verlag GmbH Germany 2017

\begin{abstract}
Summary: Myeloperoxidase (MPO) is released from activated neutrophils. The inflammation in preeclampsia was found to be associated with endothelial dysfunction. We hypothesized that cardiac and circulating MPO levels are elevated in hypertensive pregnancy. Systolic and diastolic blood pressure and heart rate were measured on pregnancy days 14, 16, 18 and 20 in normal pregnant and hypertensive pregnant rats. Left and right ventricle weights, the number of viable fetuses, litter size, fetal and placenta weights were recorded on gestational day 21. Circulating and cardiac MPO activities, soluble fms-like tyrosine kinase-1 (sFlt-1) and vascular endothelial growth factor (VEGF) and nitric oxide (NO) were detected. The results showed increases in cardiac (left, but not right ventricle) and circulating MPO activities, and concomitantly lower number of viable fetuses, litter size, and fetal and placenta weights, and decreases in NO in hypertensive pregnant rats. Also, the increases in circulating sFlt-1 and VEGF were found in hypertensive pregnant group. In conclusion, maternal and fetal detrimental changes along with increases in circulating sFlt-1 and VEGF in hypertensive pregnancy may be associated with increases in cardiac and circulating MPO activities, confirming the causative role of inflammatory response in preeclampsia.
\end{abstract}

Key words: hypertensive pregnancy; preeclampsia; rats; myeloperoxidase

Healthy pregnancy has been associated with systemic inflammation, which may be exacerbated in preeclampsia, an important pregnancy disorder characterized by hypertension and associated with elevated levels of soluble fms-like tyrosine kinase-1 (sFlt-1) after the 20th week of gestation ${ }^{[1]}$. A greater number of neutrophils in the vasculature were found in preeclamptic women than in those with normal pregnancies ${ }^{[2,3]}$. Also, the increase in the number of leukocytes during preeclampsia is markedly accompanied with the increases in the markers of leukocytes activation ${ }^{[3,4]}$. Increased neutrophil activation has been shown in hypertension in pregnancy ${ }^{[5]}$ and neutrophil depletion attenuates the placental ischemiainduced hypertension in pregnant rats ${ }^{[6]}$.

Neutrophils and monocytes normally produce and release myeloperoxidase (MPO). MPO reflects the degree of neutrophil activation and it has been associated with vascular dysfunction and pathophysiology of vascular inflammatory diseases, including coronary artery disease ${ }^{[7,8]}$. Indeed, patients with coronary artery disease have presented elevated circulating levels of MPO, which is now considered as a new biomarker of inflam-

Ming-lin ZHU, E-mail: zhuminglin@whu.edu.cn

${ }^{\#}$ Corresponding authors, Jin-ping ZHAO, E-mail: jpzhao@ tjh.tjmu.edu.cn, Carlos A. Dias-Junior, E-mail: carlosjunior@ ibb.unesp.br

*This study was supported by the Fundacao de Am-paro a Pesquisa do Estado de Sao Paulo (FAPESP, Brazil). mation in ischemic heart disease and acute coronary syndromes ${ }^{[9,10]}$. Also, elevated levels of MPO have been observed in the placenta and circulation of women with preeclampsia ${ }^{[11]}$, suggesting that increased levels of MPO may be related to endothelial dysfunction in hypertensive pregnancy ${ }^{[11,12]}$. Once in the systemic circulation, MPO may remain sequestered in the subendothelial space, accumulating in the endothelial cell matrix ${ }^{[13]}$, and MPO catalytically consumes nitric oxide $(\mathrm{NO})$ as a substrate, limiting its bioavailability and function ${ }^{[14]}$. In agreement, in vitro and animal studies have demonstrated that after leukocyte degranulation, MPO localized in and around vascular endothelial cells impaires endothelium-dependent relaxant responses of $\mathrm{NO}^{[14,15]}$.

Taken together, the above findings suggest that over-activity of MPO in the circulation of women with preeclampsia may increase the risk for cardiovascular disease after delivery, and it is related to many metabolic disturbances, including an enhanced systemic inflammatory response and endothelial dysfunction ${ }^{[16]}$. However, the specific role of MPO during preeclampsia remains unclear, and surprisingly no previous study had examined the cardiac MPO activity in hypertension in pregnancy. Therefore, we hypothesized that the deleterious (maternal and fetal) changes of hypertension in pregnancy would be associated with high levels of MPO in the heart and in the circulation. 


\section{MATERIALS AND METHODS}

\subsection{Experimental Design}

Wistar rats (210-260 g) were housed in cages at $22 \pm 2^{\circ} \mathrm{C}$ on a 12 -h light/dark cycle and given free access to water and rat chow. Each pregnant rat was separately housed overnight. Day 0 of pregnancy was defined as the day when spermatozoa were found in a vaginal smear.

On pregnancy day 14 , rats were randomly separated into two groups ( $n=12$ per group, total of 24 rats): normal pregnant group, in which the pregnant rats received daily intraperitoneal (i.p.) injections of $0.9 \%$ saline solution; hypertensive pregnant group, in which the rats received i.p. injections of $\mathrm{N}$ (omega)-nitro-Larginine methyl ester (L-NAME; Sigma, USA, catalogue\# 5751) $60 \mathrm{mg} / \mathrm{kg} /$ daily $^{[17-19]}$. The rat model of hypertension in pregnancy adopted here has been widely used to examine the maternal and fetal manifestations of preeclampsia. Rats were euthanized on gestational day 21 under overdose of isoflurane followed by exsanguination. Blood samples were collected in an EDTAcoated tube (EDTA, Vacuntainer Becton-Dickinson, UK) for plasma separation. Plasma was stored at $-80^{\circ} \mathrm{C}$ until use for biochemical analysis. Heart was removed for the weight measurements and assessment of cardiac MPO activity.

All procedures for animal experimentation were approved by the Ethics Committee, Biosciences Institute of Botucatu, State University of Sao Paulo (Protocol \#619/2014), which complied with international guidelines of the European Community for the use of experimental animals.

\subsection{Blood Pressure and Heart Rate Measurements}

Systolic and diastolic blood pressure $(\mathrm{mmHg})$ and heart rate were measured on gestational days $14,16,18$ and 20, using tail-cuff plethysmography (Insight, Ribeirao Preto, Brazil, catalogue \#EFF-306), as previously described $^{[18,19]}$. Briefly, all pregnant rats were first acclimated in a quiet room, conditioned and restrained for 5-10 min in a warm box (Insight, Ribeirao Preto, Brazil, catalogue \#EFF-307), measured for 3 days for their systolic blood pressure before the pregnancy day 14 (data discarded) and then the baseline systolic blood pressure was determined as the average of the cuff inflation-deflation (3 to 6) cycles by a trained operator on pregnancy day 14 , and the mean of three measurements was recorded.

\subsection{Effects on Placenta and Fetuses}

On gestational day 21, after euthanasia, animals were placed in a supine position and cesarean section was performed. The number of viable fetuses, litter size, and fetal and placental weights were recorded. Viable fetuses were determined to be those that showed no macroscopical sign of malformation and could apparently have a normal outcome with the progression of the pregnancy, as previously reported ${ }^{[20]}$

\subsection{Determination of MPO activity}

MPO is an enzyme contained in the granules of neutrophils. Circulating plasma and tissue levels of MPO reflect the degree of neutrophil activation and the inflammatory respons $\mathrm{e}^{[21,22]}$. Briefly, $30 \mu \mathrm{L}$ of centri- fuged plasma samples were added with $100 \mu \mathrm{L}$ of tetramethylbenzidine (TMB) and $0.04 \% \mathrm{H}_{2} \mathrm{O}_{2}$. Afterwards, the microplate was incubated for $10 \mathrm{~min}$ at $37^{\circ} \mathrm{C}$, protected from light. The reaction was stopped with 100 $\mu \mathrm{L} \mathrm{H}_{2} \mathrm{SO}_{4}(1 \mathrm{~mol} / \mathrm{L})$ and the absorbance $(A)$ at $450 \mathrm{~nm}$ with correction to $630 \mathrm{~nm}$ (was read on the spectrophotometer (Synergy 4, BIOTEK, USA). The results were expressed in $\Delta A_{630} /(\mathrm{min} \cdot \mathrm{mL})$.

Hearts (left and right ventricle) were weighted and tissue samples suspended in $1 \mathrm{~mL}$ of $50 \mathrm{mmol} / \mathrm{L}$ phosphate buffer incorporating $0.5 \%$ hexadecyltrimethylammonium bromide ( $\mathrm{pH} \mathrm{6.0)}$, and minced with scissors for $15 \mathrm{~s}$ on an ice-cold plate. The resultant suspension was diluted to a final 1:20 (w/v) ratio and homogenized for $1 \mathrm{~min}$ with an automatic homogenizer (Bio-Gen PRO200 Handheld homogenizer, USA), then sonicated for $10 \mathrm{~s}$ and subjected to three freeze-thaw cycles. The homogenates were then centrifuged at $7000 \times \mathrm{g}$ and $4^{\circ} \mathrm{C}$ for $10 \mathrm{~min}$, and the supernatants were assayed for MPO activity. The results were expressed in $\Delta A_{630} /(\mathrm{min} \cdot \mathrm{mL})$.

\subsection{Determination of sFlt-1 and VEGF}

Commercial enzyme-linked immunosorbent assay (ELISA) kits for sFlt-1 (R\&D Systems Inc, USA, catalogue \#MVR100) and VEGF (R\&D Systems Inc, USA, catalogue \#RRV00) were used to determine plasma levels. Assays were performed according to manufacturer's instructions. Plasmatic levels of sFlt-1 and VEGF were expressed in $\mathrm{pg} / \mathrm{mL}$.

\subsection{Measurement of Plasma Nitrite/Nitrate (NOx) Concentrations}

The plasma NOx concentrations were determined in duplicate by using the Griess reaction, as previously described $^{[23]}$. Briefly, $40 \mu \mathrm{L}$ of plasma was incubated with the same volume of nitrate reductase buffer $(0.1$ $\mathrm{mol} / \mathrm{L}$ potassium phosphate, $\mathrm{pH} 7.5$, containing 1 $\mathrm{mmol} / \mathrm{L} \beta$-nicotinamide adenine dinucleotide phosphate and $2 \mathrm{U}$ of nitrate reductase $/ \mathrm{mL}$ ) in individual wells of a 96-well plate. Samples were allowed to incubate overnight at $37^{\circ} \mathrm{C}$ in the dark; $8 \mu \mathrm{L}$ of freshly prepared Griess reagent (1\% sulfanilamide, $0.1 \%$ naphthylethylenediamine dihydrochloride in $5 \%$ phosphoric acid) was added to each well and the plate was incubated, for 15 additional min, at room temperature. A standard nitrate curve was obtained by incubating sodium nitrate $(0.2-200 \mu \mathrm{mol} / \mathrm{L})$ with the same reductase buffer. The NOx levels in plasma were expressed in $\mu \mathrm{mol} / \mathrm{L}$.

\subsection{Statistical Analysis}

With the aid of a commercially available statistical software (GraphPad Prism® ${ }^{\circledR} 6.0$ for Windows, CA), a Shapiro-Wilk test was applied to verify the normality of data distribution. Systolic blood pressure measurements were submitted to a two-way analysis of variance (ANOVA) with time and hypertension defined as main effects, followed by Tukey's test for multiple comparisons among groups, or by a Dunnett's test to compare measurements on pregnancy day 16, 18 and 20. Paired $t$ tests were used to compare fetal and placental changes or MPO, sFlt-1, VEGF and NOx levels. Statistical significance was considered at $P<0.05$. All values are expressed as $\bar{x} \pm s \bar{x}$. 


\section{RESULTS}

\subsection{MPO Activity}

The levels of MPO in both plasma and left ventricle were significantly higher in hypertensive [pregnant group than in normal pregnant group (plasma: $2.06 \pm 0.09$ vs. $1.11 \pm 0.19 \Delta A_{630} /(\min \cdot \mathrm{mL})$; left ventricle: $0.26 \pm 0.03$ vs. $0.16 \pm 0.01 \Delta A_{630} /(\mathrm{min} \cdot \mathrm{mL})$; both $P<0.05$, fig. $1 \mathrm{~A}$ and $1 \mathrm{~B})$. However, no significant difference was observed in MPO levels in the right ventricle between hypertensive pregnant and normal pregnant groups $(0.37 \pm 0.05$ and $0.47 \pm 0.06$, respectively, fig. $1 C)$. Moreover, no significant differences were observed in total heart $(0.99 \pm 0.05$ vs. $0.97 \pm 0.05 \mathrm{~g})$, left ventricle $(0.32 \pm 0.03$ vs. $0.38 \pm 0.07 \mathrm{~g})$ and right ventricle $(0.13 \pm 0.02$ vs. $0.16 \pm 0.02 \mathrm{~g})$ weight between the two groups, respectively $(P>0.05$, fig. $1 \mathrm{D}, 1 \mathrm{E}$ and $1 \mathrm{~F})$.
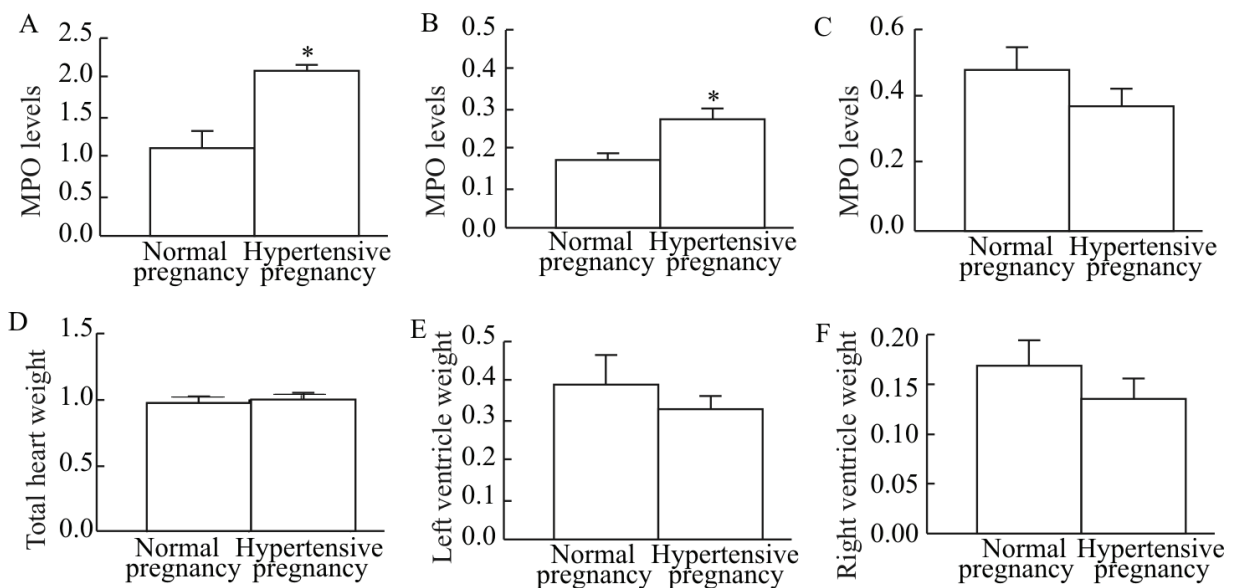

Fig. 1 Circulating (A) and cardiac MPO activity in left (B) and right (C) ventricles; and total heart (D), left (E) and right (F) ventricle weights in normal pregnant and hypertensive pregnant groups

Data are shown as $\bar{x} \pm s x .{ }^{*} P<0.05 v s$. normal pregnant group

\subsection{Blood Pressure and Heart Rate}

There were no significant differences in the systolic blood pressure values (116 and $121 \pm 3 \mathrm{mmHg}$ ) between the two groups on day 14 ( $P>0.05$, fig. 2A). Systolic blood pressure values were elevated in the hypertensive pregnant group on days 16, 18 and 20 $(154 \pm 9,148 \pm 4$ and $139 \pm 2 \mathrm{mmHg}$, respectively, $P<0.05$, fig. 2A) as compared with normal pregnant group, which presented no significant changes on day 16 $(116 \pm 1 \mathrm{mmHg})$, day $18(115 \pm 5 \mathrm{mmHg})$ and day 20 $(112 \pm 7 \mathrm{mmHg}$ ) in normal pregnant group (fig. 2A). Diastolic blood pressure was not significantly different between the two groups at days 14, 16, 18 and 20 (fig. 2B). Heart rate was decreased in hypertensive pregnant group on gestational day 16 . However, no statistically significant difference was reached (fig. 2C).

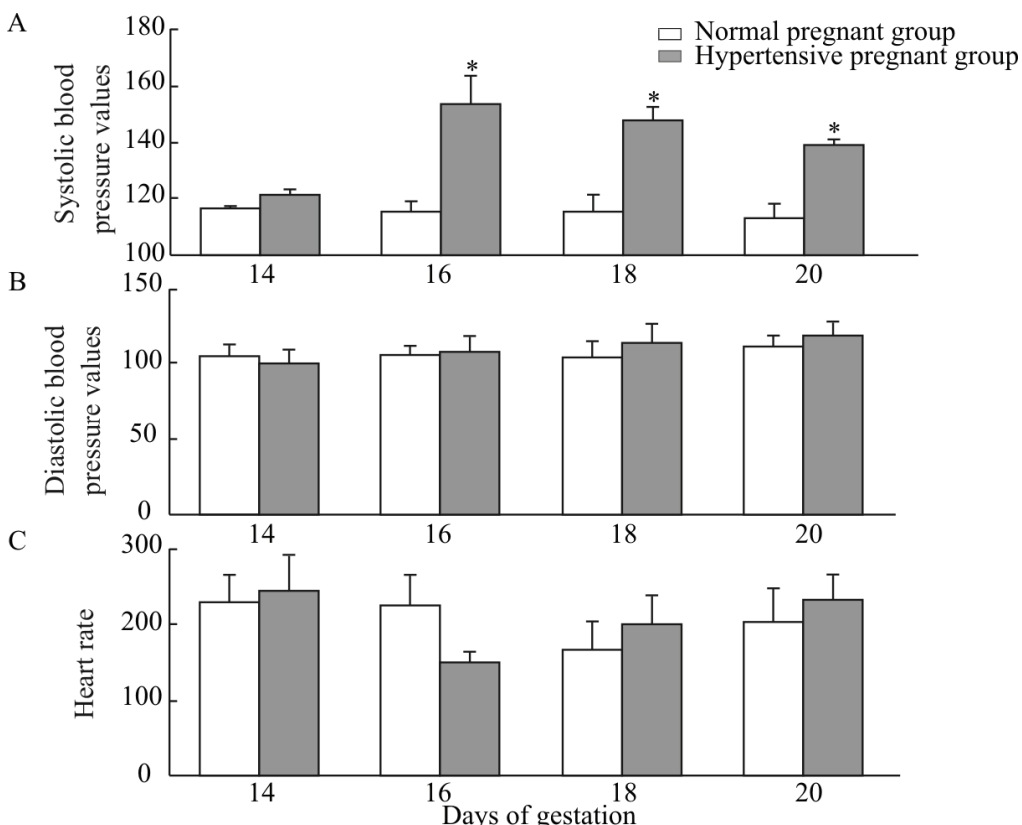

Fig. 2 Systolic (A) and diastolic (B) blood pressure and heart rate (C) measurements on days 14, 16, 18 and 20 of gestation in normal pregnant and hypertensive pregnant groups Data are shown as $x \pm s \bar{x} .{ }^{*} P<0.05 v s$. normal pregnant group 


\subsection{Placental and Fetal Weight, Number of Viable Fetuses, Litter Size}

The placental weight was profoundly reduced in hypertensive pregnant group $(0.58 \pm 0.01 \mathrm{~g})$ as compared with normal pregnant group $(0.67 \pm 0.01 \mathrm{~g}, P<0.05$, fig. $3 \mathrm{~A})$. The fetal weight was significantly lower in hypertensive pregnant group $(3.31 \pm 0.10 \mathrm{~g})$ than in normal pregnant group ( $3.86 \pm 0.06, P<0.05$, fig. 3B). The number of viable fetuses was significantly less in hypertensive pregnant group $(8.5 \pm 0.6)$ than in normal pregnant group (11.2 $\pm 0.2, P<0.05$, fig. $3 \mathrm{C})$. Also, significantly, decreased litter size was found in hypertensive pregnant group $(7 \pm 0.7)$ as compared with normal pregnant group (10.6 $\pm 0.9, P<0.05$, fig. 3D).
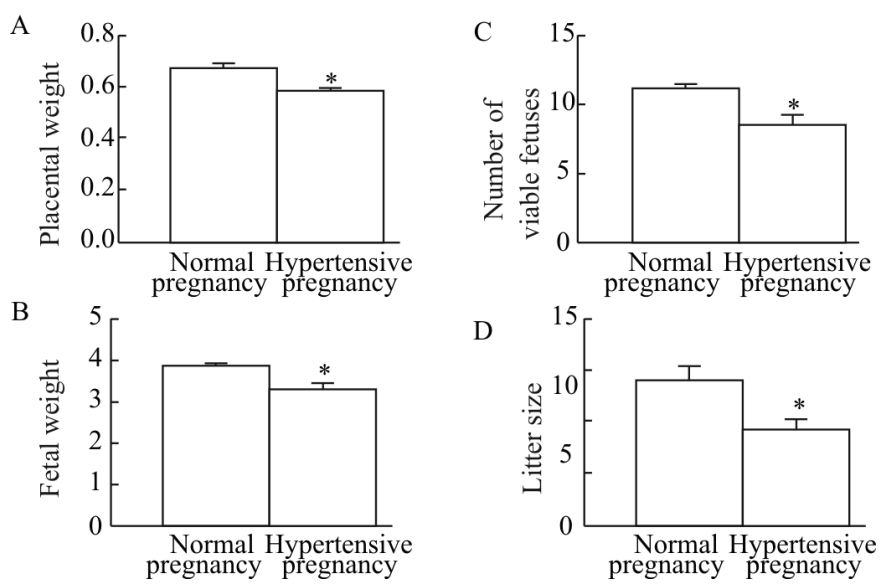

Fig. 3 Placental (A) and fetal (B) weights, and the number of viable fetuses (C) and litter size (D) in normal pregnant and hypertensive pregnant groups.

Data are shown as $\bar{x} \pm s \bar{x} .{ }^{*} P<0.05$ vs. normal pregnant group

\section{4 sFlt-1, VEGF and NOx Levels}

The free sFlt-1 and VEGF levels were significantly higher in hypertensive pregnant group than in normal pregnant group (free sFlt-1: $368 \pm 54$ vs. $162 \pm 4$ pg/mL; VEGF: $77 \pm 4$ vs. $58 \pm 6 \mathrm{pg} / \mathrm{mL}$, both $P<0.05$, fig.
4A and 4B). Also, lower NOx concentrations in plasma were found in hypertensive pregnant group $(31 \pm 3$ $\mu \mathrm{mol} / \mathrm{L})$ than in normal pregnant group $(41 \pm 4 \mu \mathrm{mol} / \mathrm{L}$, $P<0.05$, fig. 4 C).
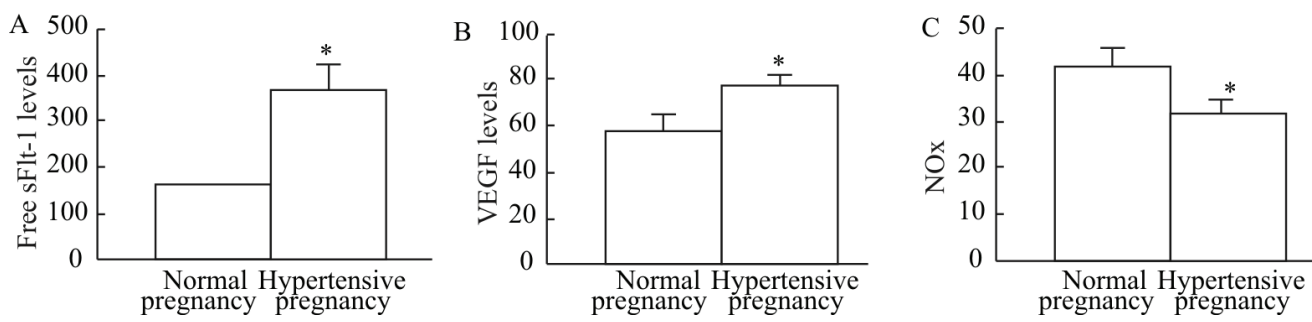

Fig. 4 sFlt-1 (A), VEGF (B) and NOx (C) levels in plasma in normal pregnant and hypertensive pregnant groups

Data are shown as $x \pm s x .{ }^{*} P<0.05$ vs. normal pregnant group

\section{DISCUSSION}

Our study demonstrated that hypertension in pregnancy induced higher MPO activity in left ventricle and in plasma than normal pregnancy, and these changes were accompanied with reductions in the number of pups (litter size) and viable fetuses, decreases in fetal and placenta weights and concomitant increases in circulating levels of sFlt-1 and VEGF and decreases in NO bioavailability.

Animal models of hypertension in pregnancy have been developed and adopted to examine the pathogenesis of preeclampsia ${ }^{[17-20,24]}$. The model of hypertension in pregnancy established in our study has been documented to be widely used to examine the maternal and fetal manifestations of preeclampsia in pregnant rats ${ }^{[17-}$ ${ }^{20]}$. The present study revealed that hypertension in pregnancy led to elevated systolic blood pressure, decreased litter size ${ }^{[25]}$ and viable fetuses ${ }^{[26]}$, and reduced fetal and placental weights ${ }^{[27]}$, which are similar to the deleterious changes seen in preeclamptic women ${ }^{[1,11]}$. The relative lag in placental weight suggests that there may be a compensatory mechanism such as enhanced placental transport capacity to meet the demands of the growing fetus and this phenomenon has been shown in other studies ${ }^{[17-28]}$. Moreover, no differences were observed in diastolic blood pressure and heart rate between the two groups. However, the heart rate tended to decrease on gestational day 16 in hypertensive pregnant group. Curiously, this trend of a lower heart rate occurred concomitantly with a significant increase in systolic blood pressure, suggesting a failed compensatory response. In accordance, previous studies reported that 
L-NAME induced an increase in systolic blood pressure and a diminished heart rate ${ }^{[29,30]}$.

To our knowledge, this is the first study showing that the cardiac MPO activity was increased in hypertension in pregnancy, which is in accordance with previous studies reporting elevations of circulating MPO levels in patients with preeclampsia ${ }^{[11,29]}$, suggesting that the most probable source of MPO is activated inflammatory cells in both heart (left ventricle) and circulation. Some study also supports this suggestion. Greer et al found neutrophils activation in hypertension in pregnancy $^{[5]}$, and neutrophil depletion could attenuate the placental ischemia-induced hypertension in pregnant rats $^{[6]}$. In addition, it has been suggested that high levels of MPO may target mainly the endothelium of women with preeclampsia, causing endothelial dysfunction in hypertension in pregnancy ${ }^{[11]}$.

Additionally, previous studies demonstrated that MPO may accumulate in the endothelial cell matrix ${ }^{[13,31]}$, thus, causing damage to the endothelium, suggesting that the elevations in cardiac and circulating MPO levels may be associated with cardiovascular events and have predictive power in high-risk patients with preeclamp$\mathrm{sia}^{[11]}$. Therefore, MPO may play a causative role in the numerous symptoms of preeclamptic women including: hypertension, systemic inflammation, and endothelial dysfunction $^{[1-3]}$.

Moreover, an important question is if the elevated MPO activity is associated with increases in circulating levels of sFlt-1 in rats with hypertension-in-pregnancy. The MPO is abundantly expressed in neutrophil granulocytes, which may also be sources of increases in circulating sFlt- $1^{[11]}$, releasing simultaneously proteases that along with sFlt-1 may cause vascular endothelial injury ${ }^{[11,21]}$. Accordingly, we found the increase in circulating sFlt-1 levels in hypertensive pregnant rats as compared with normal pregnancies. So, together, our findings are in accordance with the notion that sFlt-1 along with MPO may be originated from circulating mononuclear cells ${ }^{[11,21]}$, causing vascular endothelial injury $^{[13]}$ and concomitantly hypertension in pregnant rats.

Furthermore, in our study, pregnant rats with hypertension presented increases in circulating levels of VEGF. Recent evidence has suggested that the diminished bioavailability of NO caused by L-NAME may induce a consistent vasoconstricted state in hypertensive pregnant animals. Given that VEGF normally acts as a vasodilator, its increase may represent an unsuccessful vascular rescue response ${ }^{[18,19,32]}$.

In addition, the reductions on the formation of NO in the present study are supported by previous results suggesting that NO may be inversely related to circulating levels of sFlt-1, emphasizing that endothelial dysfunction during preeclampsia may be exaggerated by reductions of NO bioavailability along with increases in levels of sFlt-1 ${ }^{[33-35]}$.

Importantly, some limitations should be taken into account in this study. Firstly, since parameters of cellular function were not evaluated in this study, more investigations are needed to clarify the role of MPO in the heart $^{[36]}$ from preeclampsia-like syndromes. Secondly, since clinical studies in obstetric patients always involve a delicate ethical issue, animal models were used to examine the pathophysiology of preeclampsia. Conclusions derived from the animal experiments should be cautiously extrapolated to human beings. Further studies are warranted to examine the MPO activities in preeclampsia women.

In conclusion, our results showed that both maternal and fetal detrimental changes (hypertension, reductions in the number of pups and viable fetuses, and lower fetal and placental weights) along with increases in circulating sFlt-1 and VEGF and decreases in NO bioavailability found in hypertension in pregnancy may be associated with increases in cardiac and circulating levels of MPO, confirming the causative role of inflammatory response in preeclampsia.

\section{Acknowledgement}

We are indebted to Valeria Cristina Sandrim and Helio Kushima for their co-operation and technique assistance provided.

\section{Conflict of Interest Statement}

There are no known conflicts of interest in this study.

\section{REFERENCES}

1 Noris M, Perico N, Remuzzi G. Mechanisms of disease: pre-eclampsia. Nat Clin Pract Nephrol, 2005,1(2):98-114

2 Luppi $\mathrm{P}$, Haluszczak C, Trucco M, et al. Normal pregnancy is associated with peripheral leukocyte activation. Am J Reprod Immunol, 2002,47(2):72-81

3 Sacks GP, Studena K, Sargent K, et al. Normal pregnancy and preeclampsia both produce inflammatory changes in peripheral blood leukocytes akin to those of sepsis. Am J Obstet Gynecol, 1998,179(1):80-86.

4 Tsukimori $\mathrm{K}$, Maeda $\mathrm{H}$, Ishida $\mathrm{K}$, et al. The superoxide generation of neutrophils in normal and preeclamptic pregnancies. Obstet Gynecol, 1993,81(4):536-540

5 Greer IA, Haddad NG, Dawes J, et al. Neutrophil activation in pregnancy-induced hypertension. British $\mathrm{J} \mathrm{Ob-}$ stetrics Gynaecol, 1989,96(8):978-982

6 Regal JF, Lillegard KE, Bauer AJ, et al. Neutrophil depletion attenuates placental ischemia-induced hypertension in the rat. PLoS One, 2015,10(7): e0132063

7 Sugiyama S, Okada Y, Sukhova GK, et al. Macrophage myeloperoxidase regulation by granulocyte macrophage colony-stimulating factor in human atherosclerosis and implications in acute coronary syndromes. Am J Pathol, 2001,158(3):879-891

8 Klebanoff SJ. Myeloperoxidase: friend and foe. J Leukocyte Biol, 2005,77(5):598-625

9 Brennan ML, Penn MS, Van Lente F, et al. Prognostic value of myeloperoxidase in patients with chest pain. New Eng J Med, 2003,349(17):1595-1604

10 Khan SQ, Kelly D, Quinn P, et al. Myeloperoxidase aids prognostication together with N-terminal pro-B-type natriuretic peptide in high-risk patients with acute ST elevation myocardial infarction. Heart, 2007,93(7):826-831 
11 Gandley RE, Rohland J, Zhou Y, et al. Increased myeloperoxidase in the placenta and circulation of women with preeclampsia. Hypertension, 2008,52(2):387-393

12 Vita JA, Brennan ML, Gokce N, et al. Serum myeloperoxidase levels independently predict endothelial dysfunction in humans. Circulation, 2004,110(9):1134-1139

13 Baldus S, Eiserich JP, Mani A, et al. Endothelial transcytosis of myeloperoxidase confers specificity to vascular ECM proteins as targets of tyrosine nitration. J Clin Invest, 2001,108(12):1759-1770

14 Eiserich JP, Baldus S, Brennan ML, et al. Myeloperoxidase, a leukocyte-derived vascular NO oxidase. Science, 2002,296(5577):2391-2394

15 Eiserich JP, Hristova M, Cross CE, et al. Formation of nitric oxide-derived inflammatory oxidants by myeloperoxidase in neutrophils. Nature, 1998,391(6665):393397

16 Ness RB, Hubel CA. Risk for coronary artery disease and morbid preeclampsia: a commentary. Ann Epidemiol, 2005,15(9):726-733

17 Yang X, Guo L, Sun X, et al. Protective effects of hydrogen-rich saline in preeclampsia rat model. Placenta, 2011,32(9):681-686

18 Possomato-Vieira JS, Gonçalves-Rizzi VH, Graça TU, et al. Sodium hydrosulfide prevents hypertension and increases in vascular endotelial growth factor and soluble fms-like tyrosine kinase-1 in hypertensive pregnant rats. Naunyn Schmiedebergs Arch Pharmacol, 2016,389(12): 1325-1332

19 Gonçalves-Rizzi VH, Possomato-Vieira JS, Sales Graça $\mathrm{TU}$, et al. Sodium nitrite attenuates hypertension-inpregnancy and blunts increases in soluble fms-like tyrosine kinase-1 and in vascular endothelial growth factor. Nitric Oxide, 2016,57:71-78

20 Ma RQ, Sun MN, Yang Z. Effects of preeclampsia-like symptoms at early gestational stage on feto-placental outcomes in a mouse model. Chin Med J (Engl), 2010,123(6):707-712

21 Suzuki K, Ota H, Sasagawa S, et al. Assay method for myeloperoxidase in human polymorphonuclear leukocytes. Analytical Biochem, 1983,132(2):345-352

22 Krawisz JE, Sharon P, Stenson WF. Quantitative assay for acute intestinal inflammation based on myeloperoxidase activity. Assessment of inflammation in the rat and hamster models. Gastroenterology,1984,87(6):1344-1350

23 Gonçalves-Rizzi VH, Nascimento RA, PossomatoVieira JS, et al. Sodium nitrite prevents both reductions in circulating nitric oxide and hypertension in 7-day lead-treated rats. Basic Clin Pharmacol Toxicol, 2016,118(3):225-230

24 Possomato-Vieira JS, Khalil RA. Mechanisms of endothelial dysfunction in hypertensive pregnancy and preeclampsia. Adv Pharmacol, 2016,77:361-431
25 Shi X, Chen P, Liu H, et al. Decitabine improves the clinical manifestations of rats with 1-NAME-induced pre-eclampsia: a potential approach to studying preeclampsia. Hypertens Pregnancy, 2015,34(4):1-10

26 Mayr AJ, Lederer W, Wolf HJ, et al. Morphologic changes of the uteroplacental unit in preeclampsia-like syndrome in rats. Hypertens Pregnancy, 2005,24(1):2937

27 Motta C, Grosso C, Zanuzzi C, et al. Effect of sildenafil on pre-eclampsia-like mouse model induced by L-Name. Reprod Domest Anim, 2015,50(4):611-616

28 Kaya A, Boztosun A, Seckin H, et al. The evaluation of hypoxia-inducible factor 1 in N-nitro-L-arginine methyl ester preeclampsia model of pregnant rats. J Investig Med, 2011,59(8):1268-1272

29 Sadek SA, Rashed LA, Bassam AM, et al. Effect of aliskiren, telmisartan and torsemide on cardiac dysfunction in L-nitro arginine methyl ester (L-NAME) induced hypertension in rats, J Adv Res, 2015,6(6):96774

30 Tomida T, Numaguchi Y, Nishimoto Y, et al. Inhibition of COX-2 prevents hypertension and proteinuria associated with a decrease of 8-iso-PGF2 formation in LNAME- treated rats, J Hypertens, 2003,21(3):601-609

31 Mellembakken JR, Aukrust P, Olafsen MK, et al. Activation of leukocytes during the uteroplacental passage in preeclampsia. Hypertension, 2002,39(1):155160

32 Bosio PM, Wheeler T, Anthony F, et al. Maternal plasma vascular endothelial growth factor concentrations in normal and hypertensive pregnancies and their relationship to peripheral vascular resistance. Am J Clin Exp Obstet Gynecol, 2001,184(2):146-152

33 Sandrim VC, Palei AC, Metzger IF, et al. Nitric oxide formation is inversely related to serum levels of antiangiogenic factors soluble fms-like tyrosine kinase-1 and soluble endogline in preeclampsia. Hypertension, 2008,52(2):402-407

34 Pimentel AM, Pereira NR, Costa CA, et al. L-argininenitric oxide pathway and oxidative stress in plasma and platelets of patients with pre-eclampsia. Hypertens Res, 2013,36(9):783-788

35 Amaral LM, Cornelius DC, Harmon A, et al. 17hydroxyprogesterone caproate significantly improves clinical characteristics of preeclampsia in the reduced uterine perfusion pressure rat model. Hypertension, 2015,65(1):225-231

36 Loria V, Dato I, Graziani F, et al. Myeloperoxidase: a new biomarker of inflammation in ischemic heart disease and acute coronary syndromes. Mediators Inflamm, 2008,2008:135 625

(Received April 12, 2017; revised June 24, 2017) 